\title{
Sintomas neuropsiquiátricos na doença de Alzheimer: frequência, correlação e ansiedade do cuidador
}

\author{
Neuropsychiatric symptoms in Alzheimer's disease: frequency, correlation and anxiety among \\ caregivers
}

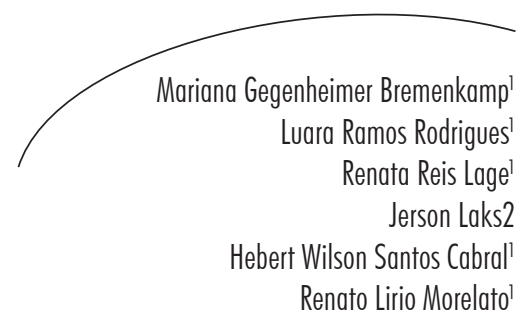

\section{Resumo}

Introdução: Sintomas neuropsiquiátricos ocorrem em 80-90\% dos pacientes com demência, interferem significativamente na autonomia do paciente e ocasionam alta carga física, financeira e emocional ao núcleo familiar, resultando em institucionalização precoce. Objetivos: Identificar sintomas neuropsiquiátricos mais frequentes e de maior gravidade nos pacientes portadores da doença de Alzheimer; especificar quais distúrbios são descritos como mais desgastantes pelo cuidador; e correlacionar os sintomas neuropsiquiátricos. Métodos: Realizou-se estudo transversal, descritivo e exploratório, durante um ano, com idosos com provável doença de Alzheimer, atendidos no ambulatório de geriatria de uma instituição filantrópica de Vitória, que estavam acompanhados do principal cuidador. As duas subescalas brasileiras do Inventário Neuropsiquiátrico foram aplicadas ao acompanhante, e os sintomas foram correlacionados utilizando a correlação de Pearson e Spearman. Resultados: Nos 50 pacientes analisados, o comportamento motor aberrante foi a manifestação mais frequente, enquanto a agitação apresentou maior gravidade entre os pacientes e foi relatada como o sintoma mais desgastante pelos cuidadores, que eram, predominantemente, filhas. Múltiplas correlações entre sintomas foram encontradas, sendo muito fortes: delírio e alucinação; delírio e ansiedade; delírio e disforia; agitação e irritabilidade. Conclusão: Os resultados reforçam a necessidade de estudos voltados para a abordagem das manifestações neuropsiquiátricas, dada sua alta prevalência e à presença de sintomas concomitantes que geram alto grau de desgaste no cuidador.

\footnotetext{
Serviço de Geriatria do Hospital Santa Casa de Misericórdia de Vitória-ES. Escola Superior de Ciências da Santa Casa de Misericórida de Vitória. Vitória, ES, Brasil.

2 Centro de Doenças de Alzheimer e outras Desordens Mentais na Velhice, Instituto de Psiquiatria. Universidade Federal do Rio de Janeiro. Rio de Janeiro, RJ, Brasil.
}

Financiamento: a Fundação de Amparo à Pesquisa do Espírito Santo (FAPES) financiou o estudo por meio do Programa Institucional de Bolsas de Iniciação Científica (PIBIC), concedido à Mariana Gegenheimer Bremenkamp, processo no 376/2012.

Palavras-chave: Doença de Alzheimer. Idoso. Sintoma Neuropsiquiátrico. 


\section{Abstract}

Introduction: Neuropsychiatric symptoms occur in $80-90 \%$ of patients with dementia, interfere significantly in the patient's autonomy and cause high physical, financial and emotional tribulation to the family nucleus, leading to early institutionalization. Objectives: Identifying neuropsychiatric symptoms more frequent and more severe in patients who are Alzheimer's disease carriers; specifying which disturbs cause more anxiety on caregivers, and correlating neuropsychiatric symptoms. Methods: Sectional, descriptive and exploratory study was conducted during one year, with elders who might have Alzheimer's disease and were attended as outpatients of a philanthropic institution in Vitoria city and were accompanied by the main caregiver. Two Brazilian subscales of the Neuropsychiatric Inventory were applied to the companions, and the symptoms were correlated using Pearson and Spearman's correlations. Results: In the 50 patients analyzed, aberrant motor behavior was the most common manifestation, while

Key words: Alzheimer's Disease. Elderly. Neuropsychiatric Symptoms agitation was more severe among patients and was reported as the worst symptom by caregivers, who were predominantly daughters. Multiple correlations were found between symptoms, being very strong: delirium and hallucination, delirium and anxiety, delirium and dysphoria, agitation and irritability. Conclusion: Results reinforce the need for studies aimed at approaching neuropsychiatric manifestations, due their high prevalence and the presence of concomitant symptoms that generate high degree of anxiety among caregivers.

\section{INTRODUÇÃO}

As manifestações neuropsiquiátricas, também denominadas sintomas comportamentais e psicológicos da demência (SCPD), se definem por um conjunto de sintomas e sinais relacionados a transtornos da percepção, do conteúdo do pensamento, do humor ou do comportamento. ${ }^{1}$ Ocorrem em $80-90 \%$ dos pacientes durante o curso da demência ${ }^{2}$ e variam de acordo com a gravidade e o subtipo da doença, afetando regiões específicas do cérebro. ${ }^{3}$

Doze são os sintomas comportamentais descritos, ${ }^{4}$ sendo que, devido à ocorrência concomitante dos distúrbios, um consórcio europeu os agrupou em quatro subsíndromes: hiperatividade (agitação, euforia, desinibição, irritabilidade e comportamento motor aberrante); psicose (alucinação, delírios e distúrbios do sono); sintomas afetivos (depressão e ansiedade); e apatia (apatia e distúrbio da alimentação). ${ }^{5}$

Os distúrbios neuropsiquiátricos são apontados como o maior problema dos pacientes com demência. ${ }^{3}$ Estão associados ao maior grau de comprometimento cognitivo e à rápida progressão da doença, diminuindo a qualidade de vida do paciente (aumentando a morbidade) e aumentando o estresse do cuidador. Além disso, aumentam os custos dos cuidados e, apesar de passíveis de intervenções terapêuticas, estão entre os mais importantes fatores de institucionalização precoce. ${ }^{3,6,7}$

Estudos elaborados no Brasil apresentaram a apatia, comportamento motor aberrante, ansiedade e depressão como os sintomas mais frequentes. ${ }^{8}$ Estudo realizado simultaneamente no Brasil e na Noruega, ${ }^{9}$ em pacientes portadores da doença de Alzheimer, demência vascular ou mista, observou que as subsíndromes psicose e sintomas afetivos foram mais frequentes. Em outro estudo, realizado na França, 75\% dos pacientes apresentavam psicose e hiperatividade. ${ }^{10}$

Referindo-se ao desgaste do cuidador, uma publicação associou a presença de delírios nos pacientes portadores de demência como causa de exaustão emocional no familiar cuidador com depressão. ${ }^{11}$ Embora os SCPDs causem significativo impacto social e econômico, 
estudos brasileiros sobre o assunto são escassos. Conhecer a frequência e gravidade dos sintomas e sua influência no desgaste do cuidador é relevante para o melhor manejo do tratamento, visando à melhoria da qualidade de vida do paciente e de seu núcleo familiar/cuidador.

Este estudo teve como objetivo identificar os sintomas neuropsiquiátricos mais frequentes e de maior gravidade entre os pacientes com doença de Alzheimer (DA); especificar quais distúrbios são descritos como mais desgastante pelo cuidador; e correlacionar os sintomas neuropsiquiátricos.

\section{METODOLOGIA}

Estudo de corte transversal, descritivo e exploratório, realizado no período de fevereiro de 2012 a fevereiro de 2013, no Hospital Santa Casa de Misericórdia de Vitória (HSCMV), durante as consultas dos idosos.

Duzentos pacientes/mês, com idade superior a 60 anos, são atendidos no ambulatório de geriatria do HSCMV. São 800 pacientes cadastrados, com retorno periódico. Há um acréscimo de 20 novos agendamentos/mês, totalizando 1.040 pacientes ao ano, sendo $10 \%$ portadores de algum grau de demência. Considerando um erro amostral de $10 \%$, poder do teste de $80 \%$ (erro tipo II) em um nível de significância de 5\% (erro tipo I), foi calculada uma amostra de 34 indivíduos. Considerando $20 \%$ de provável perda, foi determinada uma amostra de 42 pacientes.

Inicialmente, foram incluídos 72 pacientes de ambos os sexos, sem distinção de raça e status socioeconômico. Vinte e dois pacientes foram excluídos por apresentarem demência degenerativa não Alzheimer, demência vascular e demência mista, restando 50 pacientes que preenchiam os critérios para o diagnóstico provável de DA, segundo o Manual Diagnóstico e Estatístico de Transtornos Mentais - quarta edição (DSM-IV) $)^{12}$ e o National Institute of Neurological and Communicative Diseases and Stroke/Alzheimer's Disease and Related Disorders Association (NINCDSADRDA), e que estavam acompanhados de seu principal cuidador, capaz de prestar informações confiáveis a respeito dos sintomas neuropsiquiátricos apresentados no último mês.

Foram excluídos, ainda, pacientes moradores de instituições de longa permanência para idosos ou que apresentavam história de transtornos psiquiátricos prévios ou concomitantes não devidos ao processo degenerativo.

O projeto foi aprovado pelo Comitê de Ética em Pesquisa da Escola Superior de Ciências da Santa Casa de Misericórdia de Vitória, sob o n ${ }^{\circ}$ 004/2012. Todos os pacientes e responsáveis que participaram da pesquisa receberam esclarecimentos sobre os objetivos do trabalho, sendo informados sobre a confidencialidade dos resultados e que teriam a privacidade garantida. Os mesmos poderiam se recusar a participar do projeto ou retirar seu consentimento a qualquer tempo. Após a concordância em participar do estudo, foi colhida a assinatura do Termo de Consentimento Livre e Esclarecido (TCLE) pelo familiar e/ou cuidador do paciente.

Instrumento de medição

Inicialmente, foram respondidas questões a respeito de informações gerais de identificação do paciente, acrescidas do nome e parentesco do informante, tempo de demência do paciente, comorbidades e medicamentos em uso. Em seguida, foram aplicados o Estadiamento Clínico das Demências (CDR), ${ }^{13}$ o Questionário de Atividades Funcionais (QAF) $)^{14}$ e o Inventário Neuropsiquiátrico (NPI) em suas duas subescalas. ${ }^{15}$

O CDR é um instrumento que determina o estágio de comprometimento funcional da demência, por meio de entrevistas semiestruturadas, com o paciente e o cuidador/ familiar, que avaliam cognição e comportamento do paciente, bem como a influência das perdas cognitivas na sua capacidade de realizar adequadamente as atividades de vida diária. Está dividido em seis categorias cognitivocomportamentais: memória, orientação, 
julgamento e resolução de problemas, relações comunitárias, atividades no lar ou de lazer e cuidados pessoais. Cada uma das seis categorias deve ser classificada em: 0 (nenhuma alteração); 0,5 (questionável); 1 (demência leve); 2 (demência moderada); e 3 (demência grave). A memória é considerada categoria principal, ou seja, de maior significado, e as demais são categorias secundárias. ${ }^{15}$ A classificação final do CDR é obtida pela análise das classificações por categorias, segundo um conjunto de regras elaboradas e validadas. ${ }^{16}$

O QAF é um questionário composto por dez perguntas, destinadas ao familiar e/ou cuidador, que avalia a dependência funcional do paciente, independentemente de sua escolaridade e nível socioeconômico, nas atividades da vida diária. Para cada atividade, o responsável pelo paciente deverá pontuar de 0 a 3, segundo o nível de comprometimento funcional: 0 - normal ou nunca o fez, mas poderia fazê-lo; 1 - fez com dificuldade ou nunca o fez e agora teria dificuldade; 2 necessita de ajuda; 3 - não é capaz. A pontuação final é obtida pela soma da pontuação atribuída a cada uma das dez atividades. Infere-se que, quanto menor a pontuação, mais independente é o indivíduo, sendo que a capacidade funcional é considerada normal se a pontuação final oscilar entre 0 e 9 pontos. Se igual ou superior a 10 pontos, pode existir uma disfunção funcional. A pontuação máxima do instrumento é de 30 pontos, indicando maior dependência..$^{15,17}$

O NPI é um instrumento confiável, validado, constituído de um questionário, administrado ao familiar e/ou cuidador, composto por perguntas estruturadas a respeito da intensidade e da frequência de manifestações neuropsiquiátricas, observadas no último mês, em pacientes com diagnóstico de demência. Originalmente desenvolvido para avaliar dez distúrbios do comportamento, ${ }^{4}$ foi modificado ${ }^{18}$ para 12 sintomas: delírios, alucinações, agitação, depressão, ansiedade, euforia, apatia, desinibição, irritabilidade, atividade motora aberrante, distúrbios do comportamento noturno e alterações do apetite. A pontuação para cada comportamento é obtida multiplicando a intensidade (1-3) pela frequência (1-4).

Além disso, uma escala auxiliar, NPI Distress (NPI-D) ${ }^{19}$ foi desenvolvida e validada para fornecer uma medida quantitativa do sofrimento experimentado pelos cuidadores em relação a cada sintoma avaliado pelo NPI apresentado pelo paciente. Para cada manifestação, o cuidador deverá graduar seu desgaste de acordo com os critérios de pontuação: 0 - nenhum desgaste; 1 - quase nada; 2 - pouco; 3 - médio; 4 - muito; 5 quase insuportável. A pontuação do NPI total é obtida a partir do somatório das duas subescalas, NPI e NPI-D. A versão brasileira das subescalas NPI e NPI-D foi validada em $2007 .^{13}$

\section{Análise estatística}

Para análise dos dados, foi empregada a estatística descritiva, sendo as variáveis categóricas descritas por percentagem; as variáveis contínuas representadas pela tendência central (média); e a variabilidade, pelo desvio-padrão.

Os sintomas neuropsiquiátricos foram submetidos a avaliação de normalidade pelo teste de Kolmogorov-Smirnov. As variáveis não paramétricas (agitação, delírio e desinibição) e paramétricas (as demais), foram correlacionadas pelo teste de Spearman e de Pearson, respectivamente.

O software SPSS 19 foi empregado para análise dos dados. Foram considerados significantes $\mathrm{p}$-valor $\leq 0,05$.

\section{RESULTADOS}

A amostra do estudo foi composta por 50 pacientes com diagnóstico provável de demência de Alzheimer; 80\% eram do sexo feminino, com média de idade de $80 \pm 5$ (65-91) anos, sendo $60 \%$ $(\mathrm{n}=30)$ viúvos, $32 \%(\mathrm{n}=16)$ casados e $8 \%(\mathrm{n}=4)$ solteiros ou divorciados. A maioria apresentava alto grau de dependência segundo a avaliação do 
QAF $(23,60 \pm 7,10)$. A análise do Estadiamento Clínico das Demências apontou que 11 (22\%) pacientes se encontravam no CDR 1; 18 (36\%) no CDR 2 e $21(42 \%)$ no CDR 3, totalizando 39 pacientes (78\%) no estágio moderado a grave da demência (CDR 2 e 3). Entre os cuidadores, houve predominância feminina ( $88 \%$ ), sendo que $66 \%(n=33)$ eram filhas; $10 \%(n=5)$, filhos; $6 \%$ $(n=3)$, netas; $6 \%(n=3)$, noras; $6 \%(n=3)$, cônjuge, e apenas um do sexo masculino; $2 \%(\mathrm{n}=1)$, irmã, $2 \%(n=1)$, sobrinha; e $2 \%$, cuidadora contratada.
De acordo com a análise dos resultados do NPI, 98\% $(n=49)$ dos pacientes obtiveram um ou mais pontos. A pontuação média foi de 35,2 pontos ( $\mathrm{d} p=21,033$; variando de 0-82). Ao ser acrescentada a subescala NPI-D, o NPI total apresentou média de 49,46 pontos ( $d p=29,542$; variando de 0-114).

A tabela 1 mostra a frequência absoluta e relativa dos pacientes que obtiveram um ou mais pontos no NPI, bem como a pontuação média para cada manifestação.

Tabela 1. Frequência de pacientes que apresentaram manifestação neuropsiquiátrica e pontuação média do NPI para cada sintoma. Vitória-ES, 2012-2013.

\begin{tabular}{l|c|c}
\hline \multicolumn{1}{c|}{ Manifestação neuropsiquiátrica } & $\mathrm{n}(\%)^{*}$ & Média do NPI (dp) \\
\hline Delírio & $18(36)$ & $2,98(4,456)$ \\
Alucinação & $24(48)$ & $3,1(4,181)$ \\
Agitação & $33(66)$ & $4,56(4,432)$ \\
Disforia & $23(46)$ & $2,76(3,799)$ \\
Ansiedade & $23(46)$ & $2,66(3,566)$ \\
Euforia & $11(22)$ & $0,8(2,01)$ \\
Apatia & $28(56)$ & $3,76(4,373)$ \\
Desinibição & $12(24)$ & $1,28(2,893)$ \\
Irritabilidade & $22(44)$ & $3,58(4,463)$ \\
Comportamento motor aberrante & $33(66)$ & $4,66(4,293)$ \\
Comportamento noturno & $22(44)$ & $2,64(3,906)$ \\
Alteração alimentar & $23(46)$ & $2,42(3,517)$ \\
\hline
\end{tabular}

$\mathrm{n}=$ frequência absoluta de pacientes que apresentaram a manifestação neuropsiquiátrica; \% $\%$ frequência relativa; dp= desvio-padrão; *inclui apenas pacientes que obtiveram um ou mais pontos no NPI.

Os sintomas com maior número de casos referidos como presentes no último mês foram agitação psicomotora e comportamento motor aberrante, com $66 \%$ dos pacientes $(n=33)$, seguidos por apatia, manifestada em $56 \%$ dos pacientes $(\mathrm{n}=28)$. Observando a média e desviopadrão do NPI de cada manifestação, destacase o comportamento motor aberrante, com
$4,66 \pm 4,293$, seguido por agitação $(4,56 \pm 4,432)$ e apatia $(3,76 \pm 4,373)$.

A frequência dos sintomas (ocasional e comum ou frequente e muito frequente), de acordo com o julgamento dos cuidadores, foi avaliada (tabela 2). O comportamento motor aberrante foi o SCPD mais frequente. 
Tabela 2. Frequência dos sintomas neuropsiquiátricos de acordo com o julgamento dos cuidadores. Vitória-ES, 2012-2013.

\begin{tabular}{l|c|c}
\hline \multicolumn{1}{c|}{ Sintoma neuropsiquiátrico } & $\begin{array}{c}\text { Ocasional ou comum } \\
\mathrm{n}(\%)\end{array}$ & $\begin{array}{c}\text { Frequente ou muito frequente } \\
\mathrm{n}(\%)\end{array}$ \\
\hline Delírio & $4(22)$ & $14(77,8)$ \\
Alucinação & $6(25)$ & $18(75)$ \\
Agitação & $8(24,3)$ & $25(74,7)$ \\
Disforia & $11(47,8)$ & $13(52,2)$ \\
Ansiedade & $10(43,5)$ & $13(56,5)$ \\
Euforia & $3(27,3)$ & $8(72,7)$ \\
Apatia & $5(17,9)$ & $23(82,1)$ \\
Desinibição & $6(50)$ & $6(50)$ \\
Irritabilidade & $8(30,8)$ & $28(69,2)$ \\
Comportamento motor aberrante & $2(6,1)$ & $31(93,9)$ \\
Comportamento noturno & $6(27,3)$ & $16(72,7)$ \\
Alteração alimentar & $4(17,3)$ & $19(82,7)$ \\
\hline
\end{tabular}

$\mathrm{n}=$ frequência absoluta dos sintomas neuropsiquiátricos; $\%=$ frequência relativa.

Observando-se o valor médio da pontuação total da gravidade para todos os sintomas, a agitação apresentou o maior valor médio $(1,48 \pm 1,119)$, seguida por comportamento motor aberrante $(1,28 \pm 1,114)$, apatia $(1,16 \pm 1,184)$, irritabilidade $(1,12 \pm 1,239)$, disforia $(1 \pm 1,161)$, alucinação $(0,96 \pm 1,160)$, ansiedade $(0,92 \pm 1,066)$, delírio $(0,9 \pm 1,199)$, comportamento noturno $(0,84 \pm 1,095)$ e alteração alimentar $(0,7 \pm 0,909)$. Euforia $(0,26 \pm 0,565)$ e desinibição $(0,52 \pm 0,995)$ tiveram as menores médias (figura 1 ).

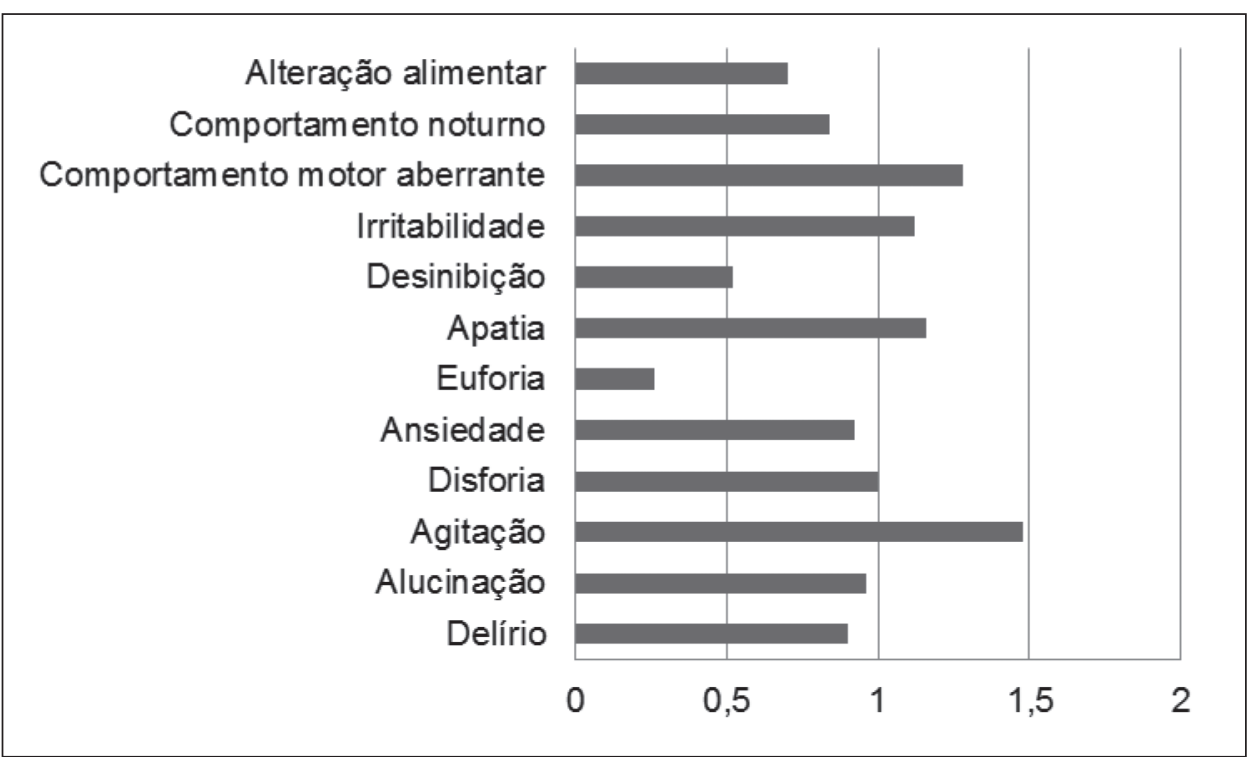

Figura 1. Média de gravidade para cada sintoma neuropsiquiátrico. Vitória-ES, 2012-2013. 
A agitação foi o sintoma descrito como mais desgastante $(1,36 \pm 1,871)$, seguida por comportamento motor aberrante $(1,64 \pm 1,838)$, irritabilidade $(1,6 \pm 1,807)$, delírio $(1,36 \pm 1,871)$, ansiedade $(1,26 \pm 1,575)$, alucinação $(1,22 \pm 1,741)$, disforia $(1,22 \pm 1,607)$, comportamento noturno $(1,14 \pm 1,552)$ e apatia $(1,12 \pm 1,466)$. Desinibição
$(0,72 \pm 1,415)$, alteração alimentar $(0,52 \pm 1,092)$ e euforia $(0,2 \pm 0,7)$ foram os sintomas mencionados como de menor desgaste (figura 2 ).

Verificou-se a correlação entre os sintomas neuropsiquiátricos, conforme ilustrado por meio da figura 3.

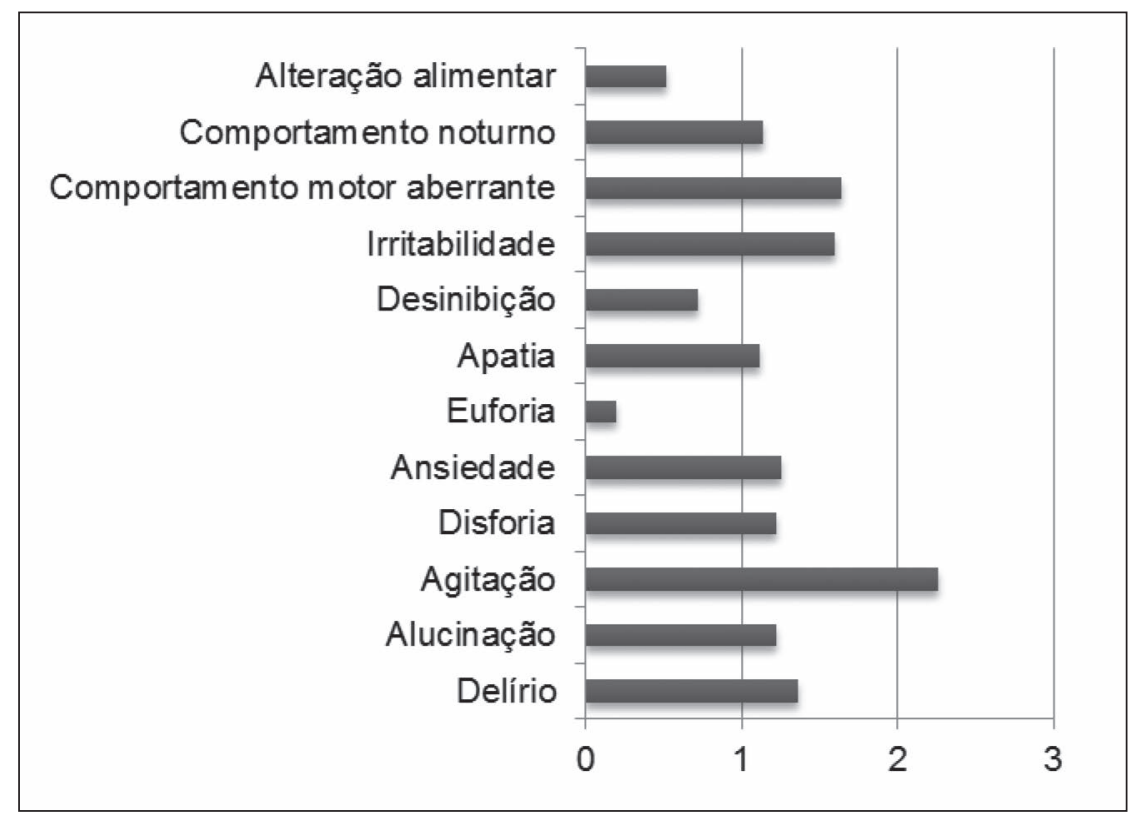

Figura 2. Média de desgaste do cuidador expresso para cada sintoma neuropsiquiátrico. Vitória-ES, 2012-2013. 


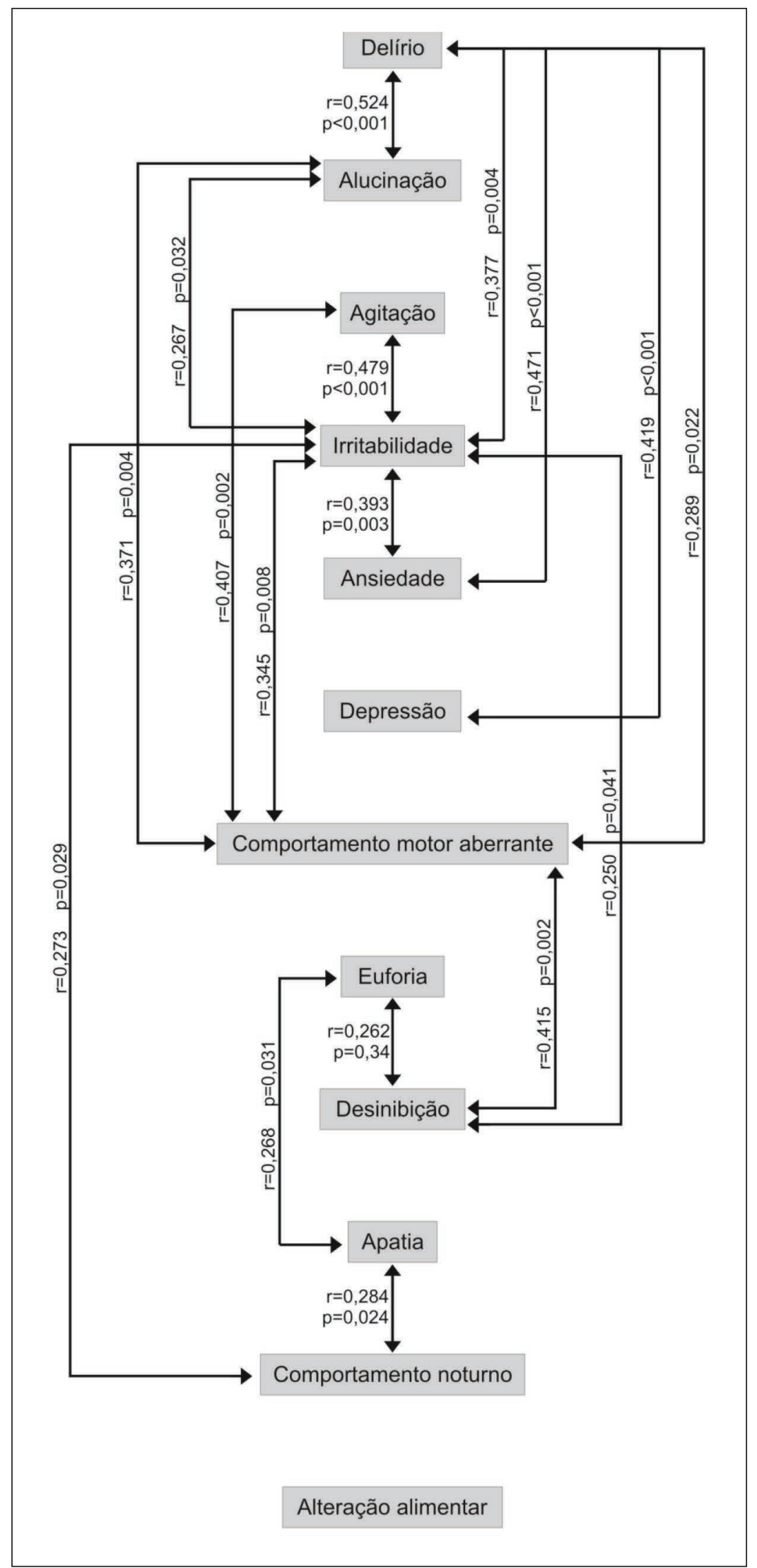

Figura 3. Correlação entre os sintomas neuropsiquiátricos. Vitória-ES, 2012-2013. 


\section{DISCUSSÃO}

Este estudo analisou a presença, no último mês, de sintomas neuropsiquiátricos em 50 pacientes com diagnóstico provável de doença de Alzheimer. As manifestações neuropsiquiátricas foram altamente frequentes, presentes em 98\% dos pacientes, fato compatível com estudos que afirmam que, em média, $90 \%$ dos pacientes desenvolvem pelo menos uma manifestação neuropsiquiátrica clinicamente relevante durante o curso da demência. ${ }^{2,20,21}$

Os sintomas mais comuns foram comportamento motor aberrante $(66 \%)$ e agitação $(66 \%)$, seguidos pela apatia $(56 \%)$, diferindo dos achados em outros estudos, que afirmaram ser a apatia o sintoma mais prevalente. ${ }^{2,9,13,22} \mathrm{~A}$ interpretação errônea durante a triagem da apatia pode ter ocorrido pela menor importância dada ao sintoma pelo cuidador, por considerá-lo como parte do estágio evolutivo da doença. Esta é, portanto, uma dificuldade observada quando o NPI é utilizado para avaliar pacientes com demência em estágio grave $(\mathrm{CDR}=3)$.

A desinibição e a euforia foram as manifestações menos comuns, como seria esperado para uma amostra de pacientes com DA. Tais sintomas são mais observados em pacientes com demência frontotemporal. ${ }^{13}$

Analisando separadamente frequência, gravidade e desgaste do cuidador, o comportamento motor aberrante foi a manifestação mais frequente, enquanto a agitação apresentou maior gravidade entre os pacientes e foi relatada como mais desgastante.

Contrariamente ao que foi reportado em estudo para validação brasileira do NPI, ${ }^{13}$ a apatia não foi citada como um dos principais sintomas desgastantes ao cuidador. No entanto, à semelhança de outros estudos, ${ }^{10} \mathrm{O}$ maior desgaste atribuído aos sintomas de hiperatividade, tais como agitação, comportamento motor aberrante e irritabilidade, é compatível com o resultado esperado, dada a alta exigência da atenção do cuidador e por ser, muitas vezes, a principal causa de necessidade de assistência médica. Pela mesma razão, era esperada maior angústia do cuidador relacionada ao comportamento noturno.

Não raro, a agitação resulta de um desconforto ou descontentamento do paciente. Os cuidadores menos treinados podem ter dificuldade em identificar que o sintoma deriva das necessidades não supridas do paciente com demência. Além do quadro demencial, diversos fatores psicológicos e ambientais, como dificuldade de comunicação com o cuidador e ambientes ruidosos, estão relacionados com o surgimento de sintomas hiperativos. $^{23}$ Desta forma, a adequada identificação e a modificação dos fatores que desencadeiam os sintomas podem reduzir a necessidade de tratamento farmacológico.

A avaliação do perfil dos cuidadores indicou resultados compatíveis com aqueles registrados por estudos que afirmam ser o principal cuidador um membro da família, geralmente do sexo feminino. ${ }^{24}$ Neste estudo, a maioria dos cuidadores eram filhas. O predomínio de mulheres em atividades voltadas ao cuidado está diretamente relacionado a aspectos culturais que atribuem essa tarefa ao gênero feminino. A necessidade de atenção integral por um único cuidador, muitas vezes determina o aumento do desgaste, por este abdicar de seu próprio cuidado e tempo de lazer em prol das exigências de atenção ao idoso.

A correlação entre as manifestações neuropsiquiátricas permitiu verificar que apenas a alteração de apetite não apresentou relação significativa com outros sintomas. As múltiplas correlações entre os sintomas estão de acordo com o esperado, uma vez que, na prática clínica, é altamente frequente a presença concomitante de mais de um sintoma neuropsiquiátrico. ${ }^{25}$

Em conformidade com estudos que tiveram por objetivo agrupar os sintomas em subsíndromes, o presente estudo identificou correlação muito forte $(\mathrm{p}<0,001)$ entre delírio e alucinação. ${ }^{2,9,26,27}$ Foi observada, ainda, correlação 
muito forte entre delírio e ansiedade; delírio e disforia; agitação e irritabilidade. Quando agrupados em subsíndromes, a correlação de mesma intensidade foi verificada no grupamento de sintomas hiperativos (agitação, irritabilidade, comportamento motor aberrante) e psicóticos (delírio e alucinação).

Este estudo apresentou algumas limitações: realizou-se análise apenas de pacientes com diagnóstico provável de doença de Alzheimer, excluindo os demais subtipos de demência devido ao pequeno tamanho da amostra. No entanto, estudos recentes têm indicado que as estimativas da prevalência de sintomas neuropsiquiátricos são semelhantes na doença de Alzheimer e demência não Alzheimer, com exceção do comportamento motor aberrante em demência do tipo Alzheimer. ${ }^{26,27}$

\section{CONCLUSÃO}

Manifestações neuropsiquiátricas estavam presentes em 98\% dos pacientes, sendo as principais no grupamento hiperativo (agitação e comportamento motor aberrante). Isoladamente, o comportamento motor aberrante foi a manifestação mais frequente, enquanto a agitação apresentou maior gravidade entre os pacientes e foi relatada como o sintoma mais desgastante pelos cuidadores, que eram predominantemente filhas. A apatia foi o terceiro sintoma mais frequente. Múltiplas correlações entre sintomas foram encontradas, sendo muito fortes: delírio e alucinação; delírio e ansiedade; delírio e disforia; agitação e irritabilidade.

A aplicação do Inventário Neuropsiquiátrico (NPI) no atendimento ambulatorial traz benefícios para a adequada avaliação dos pacientes. Entretanto, devido à extensão do questionário, seu emprego se torna praticamente inviável. Este pode ser um dos motivos para a escassez de estudos da espécie.

Conclui-se que os resultados deste trabalho reforçam a necessidade de realizar estudos voltados para a abordagem das manifestações neuropsiquiátricas, dada sua alta prevalência em todos os estágios de gravidade da doença, bem como a presença de múltiplas manifestações concomitantes, que geram alto grau de desgaste do cuidador.

\section{AGRADECIMENTOS}

À Fundação de Amparo à Pesquisa do Espírito Santo (FAPES), por financiar o estudo, e ao CNPq, pelo apoio ao Dr. Jerson Laks.

\section{REFERÊNCIAS}

1. Caramelli P, Bottino CMC. Tratando os sintomas comportamentais e psicológicos da demência (SCPD). J Bras Psiquiatr 2007;56(2):83-7.

2. Aalten P, De Vugr ME, Jaspers N, Jolles J, Verhey FRJ. The course of neuropsychiatric symptoms in dementia. Part I: findings from the two-year longitudinal Maasbed study. Int J Geriatr Psychiatry 2005;20:523-30.

3. Srikanth S, Nagaraja AV, Ratnavalli E. Neuropsychiatric symptoms in dementia frequency, relationship to dementia severity and comparison in Alzheimer's disease, vascular dementia and frontotemporal dementia. J Neurol Sci 2005;236(1-2):43-8.
4. Cummings JL, Mega M, Gray k, RosembergThompson S, Carusi DA, Gornbein J. The Neuropsychiatric Inventory: comprehensive assessment of psychopathology in dementia. Neurology 1994;44(12):2308-14

5. Aalten P, Verhey FR, Boziki M, Bullock R, Byrne EJ, Camus V, et al. Neuropsychiatric syndromes in dementia. Results from the European Alzheimer's Disease Consortium: part I. Dement Geriatr Cogn Disord 2007;24(6):457-63.

6. Vega UM, Marinho V, Engelhardt E, Laks J. Sintomas neuropsiquiátricos nas demências: relato preliminar de uma avaliação prospectiva em um ambulatório do Brasil. Arq Neuropsiquiatr 2007;65(2b):498-502. 
7. Youn JC, Lee DY, Jhoo JH, Kim KW, Choo IH, Woo JI. Prevalence of neuropsychiatric syndromes in Alzheimer's disease (AD). Arch Gerontol Geriatr 2011;52(3):258-63.

8. Tatsch, MF, Bottino CMC, Azevedo D, Hototian SR, Moscoso MA, Folquitto JC, et al. Neuropsychiatric symptoms in Alzheimer disease and cognitively impaired, nondemented elderly from a communitybased sample in Brazil: prevalence and relationship with dementia severity. Am J Geriatr Psychiatry 2006;14(5):438-45.

9. Truzzi A, Ulstein I, Valente L, Engelhardt E, Coutinho ES, Laks J, et al. Patterns of neuropsychiatric sub-syndromes in brazilian and norwegian patients with dementia. Int Psychogeriatr 2013;25(2):228-35.

10. Mézière A, Blachier M, Thomas S, Verny M, Herbaud $\mathrm{S}$, Bouillanne $\mathrm{O}$, et al. Neuropsychiatric symptoms in elderly patients: a multicenter cross-sectional study. Dement Geriatr Cogn Disord Extra 2013;3(1):123-30.

11. Truzzi A, Valente L, Ulstein I, Engelhardt E, Laks J, Engedal K. Burnout in familial caregivers of patients with dementia. Rev Bras Psiquiatr 2012;34(4):405-12.

12. American Psychiatric Association. Diagnostic and Statistical Manual of Mental Disorders (DSM-IV). 4th ed. Washington, DC: American Psychiatric Association; 1994.

13. Camozzato AL, Kochhann R, Simeoni C, Konrath CA, Pedro Franz A, Carvalho A, et al. Reliability of the brazilian portuguese version of the Neuropsychiatric Inventory (NPI) for patients with Alzheimer's disease and their caregivers. Int Psychogeriatr 2008;20(2):383-93.

14. Macedo Montaño MBM, Ramos LR. Validade da versão em português da Clinical Dementia Rating. Rev Saúde Pública 2005;39(6):912-17.

15. Pfeffer RI, Kurosaki TT, Harrah CH Jr, Chance JM, Filos S. Measurement of functional activities in older adults in the community. J Gerontol 1982;37(3):323-9.

16. Morris JC. The Clinical Dementia Rating (CDR): current version and scoring rules. Neurology 1993;43(11):2412-4.

17. Castro SD, Silva DJ, Nascimento ESR, Christofoletti G, Cavalcante JES, Lacerda MCC, et al. Alteração de equilíbrio na doença de Alzheimer: um estudo transversal. Rev Neurocienc 2011;19(3):441-8.

18. Cummings JL. The Neuropsychiatric Inventory: assessing psychopathology in dementia patients. Neurology 1997;48(Suppl 6):S10-16.

19. Kaufer DI, Cummings JL, Christine D, Bray T, Castellon S, Masterman D, et al. Assessing the impact of neuropsychiatric symptoms in Alzheimer's disease: the Neuropsychiatric Inventory Caregiver Distress Scale. J Am Geriatr Soc 1998;46(2):210-5.

20. Devanand DP, Jacobs DM, Tang MX, Del Castillo-Castaneda C, Sano M, Marder K,et al. The course of psychopathologic features in mild to moderate Alzheimer disease. Arch Gen Psychiatr 1997;54(3):257-63.

21. Mega MS, Cummings JL, Fiorello T, Gornbein J. The spectrum of behavioural changes in Alzheimer's disease. Neurology 1996;46(1):130-5.

22. Baiyewu O, Smith-Gamble V, Akinbiyi A, Lane $\mathrm{KA}$, Hall KS, Ogunniyi A, et al. Behavioral and caregiver reaction of dementia as measured by the Neuropsychiatric Inventory in nigerian community residents. Int Psychogeriatr 2003;15(4):399-409.

23. Gitlin LN, Kales HC, Lyketsos CG. Nonpharmacologic management of behavioral symptoms in dementia. JAMA 2012;308(19):2020-29.

24. Ikeda M, Fukuhara R, Shigenobu K, Hokoishi K, Maki N, Nebu A, et al. Dementia associated mental and behavioural disturbances in elderly people in the community; findings from the first Nakayama study. J Neurol Neurosurg Psychiatry 2004;75(1):146-8.

25. Gauthier S, Cummings J, Ballard C, Brodaty H, Grossberg G, Phillippe R, et al. Management of behavioral problems in Alzheimer's disease. Int Psychogeriatr 2012;22(3):346-72.

26. Lyketsos CG, Lopez O, Jones B, Fitzpatrick AL, Breitner J, DeKosky S. Prevalence of neuropsychiatric symptoms in dementia and mild cognitive impairment: results from the cardiovascular health study. JAMA 2002;288(12):1475-83.

27. Lyketsos CG, Steinberg M, Tschanz JT, Norton MC, Steffens DC, Breitner JC. Mental and behavioural disturbances in dementia: findings from the Cache County Study on Memory in Aging. Am J Psychiatr 2000;157(5):708-14. 